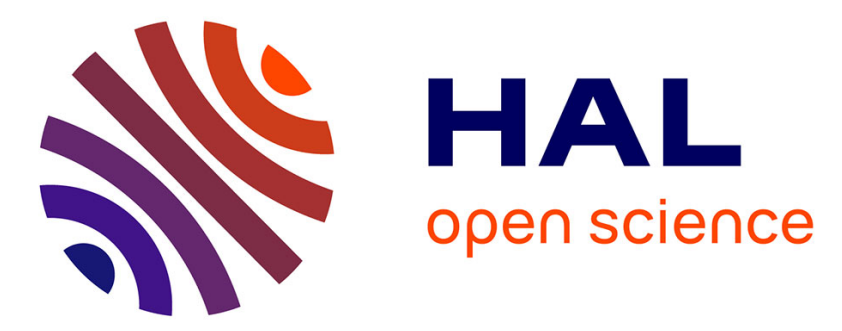

\title{
Urban morpho-types classification from SPOT-6/7 imagery and Sentinel-2 time series
}

Arnaud Le Bris, Nesrine Chehata

\section{To cite this version:}

Arnaud Le Bris, Nesrine Chehata. Urban morpho-types classification from SPOT-6/7 imagery and Sentinel-2 time series. 2019 Joint Urban Remote Sensing Event (JURSE), May 2019, Vannes, France. pp.1-4, 10.1109/JURSE.2019.8808988 . hal-02928739

\section{HAL Id: hal-02928739 \\ https://hal.science/hal-02928739}

Submitted on 2 Sep 2020

HAL is a multi-disciplinary open access archive for the deposit and dissemination of scientific research documents, whether they are published or not. The documents may come from teaching and research institutions in France or abroad, or from public or private research centers.
L'archive ouverte pluridisciplinaire HAL, est destinée au dépôt et à la diffusion de documents scientifiques de niveau recherche, publiés ou non, émanant des établissements d'enseignement et de recherche français ou étrangers, des laboratoires publics ou privés. 


\section{Urban morpho-types classification from SPOT-6/7 imagery and Sentinel-2 time series}

\author{
Arnaud Le Bris \\ Univ. Paris-Est, LASTIG STRUDEL, IGN, ENSG \\ Saint-Mande, France \\ arnaud.le-bris@ign.fr
}

\author{
Nesrine Chehata \\ EA G\&E Bordeaux INP, Bordeaux Montaigne University \\ Pessac, France \\ nesrine.chehata@ipb.fr
}

\begin{abstract}
This paper aims at detecting several urban morphotype classes out of complementary SPOT-6/7 imagery and Sentinel-2 time series and using existing Urban Atlas database. A bottom-up strategy is proposed. It first detects basic urban objects (buildings, roads, vegetation), that are used to calculate multi-scale morphological features. These features are then fed to a Random Forest classifier trained from samples out of Urban Atlas urban classes. Obtained results are optionally merged with a Random Forest classification based on Sentinel-2 time series. Obtained results are promising.

Index Terms-Classification, SPOT, Sentinel-2, Urban land-
\end{abstract} scape, Urban morpho-types, Urban areas, Urban Atlas

\section{INTRODUCTION - CONTEXT}

\section{A. Needs for different urban classes}

Artificialized areas are a key land cover and land use components [12]. They have a strong impact on the energetic budget (as for the urban heat island effect), but also on natural ecosystems (fragmentation of land cover and ecological areas) [11] and on hydrological systems (modifying water run-off in urban and peri-urban areas) [13]. Thus, knowledge about urban patterns is important to monitor urban sprawl and densification across time, providing inputs both to socioeconomic analysis and to hydrological and meteorological models [9]. Many works have already considered supervised classification to classify several urban patterns (or morphotypes). However proposed methods are very different (input data, output classes), limiting the possibility to compare these different studies [14].

Several works have focused on retrieving local climate zones [9], [10], using either high or medium resolution data or very high resolution ones. The first approaches generally perform a supervised classification directly [1], while the latter are more specific [4], [6], [7], deriving urban morphotypes out of detected urban objects. Most of them benefit from a predefined skeleton with predefined units (grid cells or blocks derived from the road network). The labeling task is processed per unit, taking into account features calculated at such a level. Current approaches require a good initialization of the land-use boundaries, in practice not known beforehand. In the present context, no predefined skeleton is available and units have to be automatically retrieved. They can have quite varying sizes and shapes depending on classes and the local context. A top-down solution has been proposed in [5], first segmenting low resolution satellite images in land-use units, and then labeling them from very high resolution images. A bottom-up approach will be preferred here.

Various urban morpho-type legends have been proposed. Some of them are quite fine and rely on Local Climate Zones [9], [10], but it is difficult to have associated reference ground truth data. The "urban" product of Theia infrastructure ${ }^{1}$ targets a more simple set of four urban classes: dense, discontinuous, industrial/commercial areas, road network. Urban Atlas product ${ }^{2}$ distinguishes four different urban density classes; isolated buildings, industrial/commercial areas, and different transportation infrastructures.

The present study aims at designing an automatic production workflow for the Theia urban product, but relying on existing Urban Atlas classes.

\section{B. Which remote sensing data?}

Urban objects generally exhibit a size smaller than $10 \mathrm{~m}$. Therefore, a higher spatial resolution than the one of Sentinel$2(10 \mathrm{~m}-20 \mathrm{~m})$ is necessary to try to delineate them and to consider sharper geometrical details. At present, Theia provides, for free, an annual coverage of SPOT-6/7 imagery over the whole French metropolitan territory. SPOT-6/7 imagery exhibits a $1.5 \mathrm{~m}$ GSD (ground sample distance) after pansharpening, which can enable the delineation of small features and the use of textural features. However, its spectral information is limited to four blue-green-red-near infrared bands. On the opposite, Sentinel-2 has a less geometric resolution but more spectral bands and an important revisit frequency (time series). Thus, these sensors are complementary and using them together would combine their advantages.

\section{PRoposed StRategy}

The proposed workflow relies on both Sentinel-2 time series and SPOT-6/7 monodate images. The fusion is performed at different steps of the method. The proposed process first extracts "topographic" elements including buildings, roads and vegetation out of SPOT-6/7 and Sentinel-2 data. Multiscale morphological features are then calculated out of the

\footnotetext{
${ }^{1}$ http://www.theia-land.fr/fr/themes/urbain

${ }^{2}$ https://www.eea.europa.eu/data-and-maps/data/copernicus-landmonitoring-service-urban-atlas
} 
masks corresponding to these classes, in order to characterize the urban patterns. A supervised classifier is trained using these features and the different urban classes of Urban Atlas. In parallel, a classifier is also trained to retrieve the same classes directly out of Sentinel-2 time series. At the end, both classifications can be merged. An overview of the method is given by Figure 1. This strategy integrates the idea that the "topographic" classification model can be trained from databases available on the whole French territory. Thus such model can be calculated locally to be adapted to various image conditions (different acquisition dates and landscapes) and to obtain classification with a similar quality everywhere. Then, a second model can be trained from this first classification to retrieve Urban Atlas classes, while Urban Atlas is only available on the most important cities.

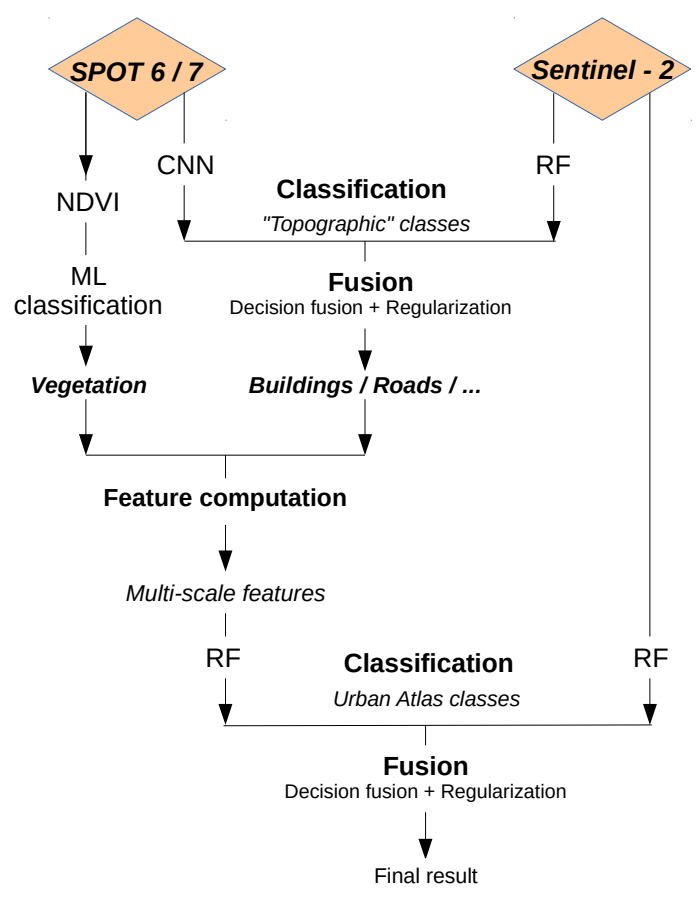

Fig. 1. Proposed workflow

\section{A. Detecting “Topographic" elements}

1) "Topographic" classification: This step consists in detecting the basic topographic elements: "buildings", "roads", "forest", "crops and other vegetation", "water" as well as an additional "around building" buffer class. The framework proposed in [3] was used for this task. Sentinel-2 time series and monodate SPOT-6/7 imagery are used within a late fusion scheme, considering original data can be classified earlier and independently by specific ad-hoc methods (Random Forest as in [1] for Sentinel-2 time series and Convolutional Neural Network for SPOT, to exploit texture information [2]). Classifiers are trained from a reference label map generated out of different topographic, forest and agricultural databases available across the whole French territory. Thus, both sources are first classified independently according to the topographic classes, generating class membership probabilities that are then merged, especially in order to extract buildings as accurately as possible.

2) Vegetation detection: The topographic classification described in previous paragraph includes two vegetation classes: "forest" and "crops and other vegetation". Unfortunately, the training areas associated to these classes are mostly located outside urban areas. As a consequence, the urban vegetation corresponding to gardens around buildings is mislabeled by this classification and included instead in the building buffer class together with other small objects. However, vegetation is a key information to characterize urban patterns and has thus to be identified. A simple yet efficient solution was adopted: a basic maximum likelihood classifier with Gaussian model for radiometric class distribution is used to detect vegetation out of the NDVI index calculated from SPOT imagery. It is trained from the original ground truth map for two classes "vegetation" (trained from forest areas) and "non vegetation" (trained from building and road samples).

\section{B. Morphological analysis}

Multi-scale morphological features are derived from the topographic class masks obtained at previous step, i.e. features are computed within different neighborhoods. Many features can be computed, but for further experiments, only the proportion of each class within a neighborhood is considered. Six different neighborhood radius are considered: $15 \mathrm{~m}, 30 \mathrm{~m}, 75$ $\mathrm{m}, 150 \mathrm{~m}, 225 \mathrm{~m}$ and $300 \mathrm{~m}$.

Multi-scale morphological features are fed to a Random Forest (RF) classifier trained from class samples from Urban Atlas database. The same idea as in [1] is used: training samples for the different urban area densities are selected out of the intersection between buildings from the BD Topo and Urban Atlas polygons.

\section{Sentinel-2 classification}

A RF classifier is also trained for a Sentinel-2 time series using the same training samples as for the SPOT imagery. Sentinel-2 is interesting since it provides a richer temporal and spectral configuration. Indeed, it offers an enhanced spectral configuration, including bands from the SWIR domain, which were shown to be useful for urban material classification, which is a key information considering the different types of urban land cover, and especially building roofs that are covered by various materials. It also enables to capture time series, or at least multitemporal images at different periods of the year. Because of its limited spatial resolution, most building pixels are not pure but include a fraction of neighboring objects, and particularly shadows. Shadows depend on the height of the masking elements (e.g. buildings) generating then and on the Sun orientation and thus on the period of the year. Therefore, these mixed urban pixels contain a latent information about shadows and thus implicitly about building heights. 


\section{SPOT and Sentinel classification fusion}

Optionally, both results can be merged within a fusion framework. In further experiments, a Dempster-Shafer rule was used.

\section{SITE AND MATERIAL}

\section{A. Study area}

The study area covers $150 \mathrm{~km}^{2}$ around Bordeaux city in South Western France. Bordeaux is the 5th biggest urban area in France, thus exhibiting different urban patterns. Besides the study area also includes rural parts and several smaller towns.

\section{B. Remote sensing data}

- Sentinel-2: 4 dates (2017-06-18, 2017-04-19, 201611-30 and 2016-09-01) along a year long time series are selected to mitigate seasonal effects on land cover classification. Only the 10 bands having a 10 or $20 \mathrm{~m}$ GSD are used and resampled to $10 \mathrm{~m}$.

- SPOT-6/7: The 4 spectral bands (blue-green-red-near infrared) pan-sharpened to $1.5 \mathrm{~m}$ of a single date (2016-03-22) acquisition are used.

\section{REsults}

Examples of obtained results are presented on Figure 2. These results are promising. At this stage, the evaluation is qualitative. One can observe that an even not perfect classification of urban topographic objects can be a basis to derive different urban morpho-types: it can be used to derive information about the different urban densities, especially to distinguish dense, discontinuous and isolated residential areas. The distinction between residential and industrial/commercial is more difficult but is favored by the urban vegetation mask detected out of SPOT imagery.

When comparing classification results with a very high resolution ortho-image, it can be noted that the dense urban center is quite well retrieved, and that even though, the different building densities of the discontinuous residential areas can be different from Urban Atlas, they generally correspond to another acceptable density class (e.g. "10$30 \%$ " instead of "30-50\%"). No density gradient effect is observed near the borders of urban areas. The most important commercial/industrial areas are generally retrieved, but smaller ones are often misclassified. Collective housing residential areas are also sometimes classified as commercial/industrial areas. Classification results using morphological features may also be different from Urban Atlas reference map since it is not up-to-date.

Obtained results were also compared to OSO land cover map [1], even though the later (based on Corine Land Cover) only distinguishes dense, discontinuous, industrial/commercial and transportation areas. Our results provide a better discrimination between dense and discontinuous urban areas, and a better detection of transportation areas.

The morphological feature based classification was also compared to a classification obtained directly from the Sentinel-2 time series. The first is more generalized thanks to the multi-scale features while the latter reflects more the variability of urban materials with for instance a discrimination of wide industrial buildings from neighboring transportation areas. Merging both classifications leads to a result where wide vegetated areas are better excluded from urban classes, industrial/commercial areas are slightly better detected, but transportation areas slightly over detected compared to original morphological feature based one.

\section{CONCLUSION AND PERSPECTIVES}

An automatic methodology extracting different urban morpho-types out of SPOT-6/7 and Sentinel-2 data was presented. Obtained results obtained for this simple method are promising.

At present, only very simple morphological features (densities) have been considered. It will be interesting to test other features (based on the distance between detected urban objects for instance).

The comparison between the morphological feature-based classification and Sentinel-2 based one is not completely fair since the latter remains a per-pixel classification. Multiscale features on different neighborhoods (mean, standard deviations) have also to be derived out of Sentinel-2 data to better apprehend the different urban densities.

Deep learning approaches should also be investigated. They could be applied to the topographic classification rather than to raw image data. Urban Atlas classes could also be replaced by building densitites calculated out of building topographic database available everywhere, contrary to Urban Atlas.

At the end, the classification results must also be smoothed in order to obtain more regular polygons (straight borders) for cartographic rendering. Building objects from "topographic"classification could be used as basis for this task.

\section{ACKNOWLEDGMENT}

This work was supported by public funds received in the framework of TOSCA project "Artificialisation et Urbanisation", funded by CNES, and GEOSUD, a project (ANR-10EQPX-20) of the program "Investissements d'Avenir" managed by the French National Research Agency.

\section{REFERENCES}

[1] J. Inglada, A. Vincent, M. Arias, B. Tardy, D. Morin, I. Rodes, "Operational High Resolution land cover map production at the country scale using satellite image time serie", Remote Sensing, vol. 9(1):95, 2017.

[2] T. Postadjian, C. Mallet, A. Le Bris, H. Sabhi, "Investigating the potential of deep neural network for large scale classification of very high resolution satellite images", ISPRS An. of Phot., Remote Sensing and Spat. Inf. Sc. , vol. 4(1/W), pp. 183-190, 2017.

[3] C. Wendl, A. Le Bris, N. Chehata, A. Puissant, "Decision fusion of SPOT-6 and Sentinel-2 images for urban area detection", IGARSS, 2018.

[4] S. Rougier, "Apport des images satellite très haute résolution spatiale couplées des donnes géographiques multi-sources pour l'analyse des espaces urbains", $\mathrm{PhD}$ thesis of Strasbourg University, 2016. 

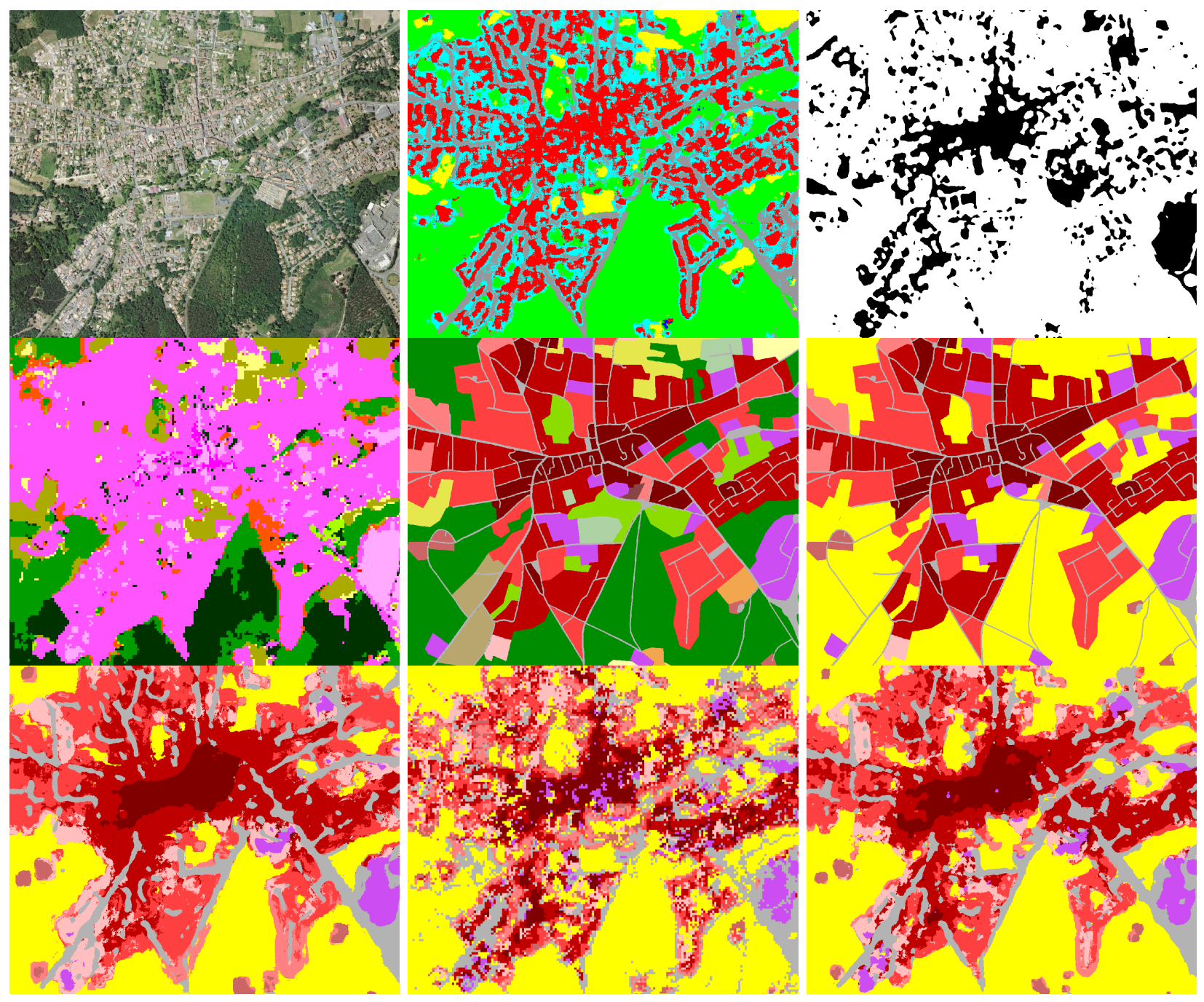

Fig. 2. First line, from left to right: ortho-image, "topographic" classification results (section II-A1) (• buildings, • roads, • forest,

, • water, • building buffer), vegetation mask detected out of SPOT images. Second line, from left to right: OSO land cover map from [1](• dense, ,- industrial/commercial, • transportation areas), Urban Atlas land cover map with all classes, Urban Atlas after aggregation of non urban classes in a single class, and aggregation of transportation areas ( $\bullet$ continuous (S.L. $>80 \%), \bullet$ discontinuous dense $(50 \%<$ S.L. $<80 \%)$, $\bullet$ discontinuous medium density $(30 \%<$ S.L. $<50 \%)$, discontinuous low density $(10 \%<$ S.L $<30 \%)$ and • discontinuous very low density (S.L. $<10 \%)$ urban fabric, • isolated structures, • industrial, commercial, public, military and private units, • aggregated transportation infrastructures, • other). Third line, from left to right: morphological features based classification (section II-B), Sentinel-2 classification, fusion result.

[5] C. Kurtz, N. Passat, P. Ganarski and A. Puissant, "Extraction of complex patterns from multiresolution remote sensing images: A hierarchical topdown methodology", Pattern Recognition, vol. 45(2), pp. 685-706, 2012.

[6] M. Li, A. Stein, W. Bijker and Q. Zhan, "Urban land use extraction from Very High Resolution remote sensing imagery using a Bayesian network", IJPRS, vol. 122, pp. 192-205, 2016.

[7] J. H. Lowry and M. B. Lowry, "Comparing spatial metrics that quantify urban form", Computers, Environment and Urban Systems, vol. 44, pp. 59-67, 2014.

[8] T. Novack, H. Kux, R.Q. Feitosa and G.A.O.P. Costa, "A knowledgebased, transferable approach for block-based urban land-use classification", IJRS, vol. 35(13), pp. 4739-4757, 2014.

[9] I.D. Stewart and T.R. Oke, "Local Climate Zones for urban temperature studies", Bulletin of the American Meteorological Society, vol. 93, pp. 1879-1900, 2012.

[10] WUDAPT: World Urban DAtabase, http://www.wudapt.org/lcz/

[11] E. G. Irwin and N. E. Bockstael, "The evolution of urban sprawl: Evidence of spatial heterogeneity and increasing land fragmentation", Proc. of the National Academy of Sciences, vol. 104(52), pp. 20672-
20677, 2007.

[12] D. Lu and Q. Weng, "Use of impervious surface in urban land-use classification", Remote Sensing of Environment, vol. 102(1-2), pp. 146160, 2006.

[13] E. Brun and L.E. Band, "Simulating runoff behavior in an urbanizing watershed", Computers, Environment and Urban Systems, vol. 24(1), pp. 5-22, 2000.

[14] E.T. Slonecker, D.B. Jennings and D. Garofalo, "Remote sensing of impervious surfaces: A review", Remote Sensing Reviews, vol. 20(3), pp. 227-255, 2001 